\title{
Caracterização das consultas abertas
}

Ana Azevedo Ramos, Inês Proença, João Magalhães, Leonor Grijó, Liliana Beirão, Maria João Canavez Peixoto, ${ }^{1}$ Sílvia Camões

\section{RESUMO}

Objetivos: A consulta aberta (CA) é um tipo de consulta fundamental no âmbito dos cuidados de saúde primários (CSP) e desempenha um papel central para a gestão eficiente de recursos, sustentabilidade e qualidade do Serviço Nacional de Saúde (SNS). Este estudo teve como principal objetivo a caracterização das CA da USF Espaço Saúde através do estudo de um conjunto alargado de variáveis, incluindo os motivos de consulta e os diagnósticos registados em cada consulta.

Tipo de estudo: Estudo transversal descritivo.

Local: USF Espaço Saúde, ACeS Grande Porto VI - Porto Ocidental.

População: Utentes que recorreram à CA da USF Espaço Saúde durante os meses de janeiro, abril e julho de 2014.

Métodos: Foi realizada a colheita de um conjunto de variáveis (idade, género, comorbilidades, nível de escolaridade, situação laboral, médico de família, médico que realizou a consulta aberta, isenção ou não-isenção de taxa moderadora, número total de consultas que o utente teve no ano de 2014, motivo da consulta aberta, diagnóstico segundo a ICPC-2 e referenciação para o SU/Hospital) através do SClínico ${ }^{\circledR}$. A análise estatística dos dados foi realizada através do programa Microsoft Excel $2015{ }^{\circledR}$. Resultados: Foram realizadas um total de $1.310 \mathrm{CA}$, representando $41,2 \%$ do total das consultas presenciais. A maioria dos utentes pertencia ao sexo feminino $(62,7 \%, n=821)$, apresentando uma média de idades de 44,8 anos. Os utentes que mais recorreram a CA foram aqueles que em relação à sua situação profissional se encontravam no ativo $(41,5 \%, n=544)$ e que eram isentos de taxas moderadoras $(58,7 \%, n=769)$. O motivo mais registado de CA foi o $A 62$ - Procedimento administrativo $(6,12 \%)$, seguindo-se o R05 - Tosse (5,35\%); o A31 e o W31 - Exame médico/avaliação de saúde parcial (ambos com 4,97\%). O diagnóstico mais registado foi o R74 - Infeção aguda do aparelho respiratório superior (6,52\%), seguido por A98 - Medicina preventiva/Manutenção da saúde (5,71\%) e K86 - Hipertensão sem complicações (5,18\%). Foi realizada referenciação para o serviço de urgência em apenas 22 consultas (1,9\%) e referenciação a consulta hospitalar em 78 consultas (6,0\%).

Conclusões: Para além da caracterização detalhada da CA realizada na USF, o estudo permitiu verificar a importância de se investir na literacia em saúde dos utentes, no sentido de compreenderem as indicações específicas que devem motivar a ida à CA. Os resultados do estudo reforçam, ainda, a importância de um correto registo clínico que, por vezes por falta de gestão de tempo dos profissionais, fica comprometido, limitando não só o seguimento clínico do utente como uma possível investigação futura. Através da análise crítica dos principais motivos/diagnósticos pelos quais os utentes recorrem à CA será possível uma melhor gestão de recursos que, aliados ao trabalho em equipa, permitirão uma melhor assistência e qualidade de resposta no âmbito das CA.

Palavras-chave: Consulta aberta; Intersubstituição médica.

\section{INTRODUÇÃO}

A medicina geral e familiar (MGF) e os cuidados de saúde primários (CSP) desempenham um papel fulcral no funcionamento de todo o sistema de saúde, tendo como objetivo a promoção da saúde, a prevenção da doença e a ligação a outros serviços de saúde, garantindo a continuidade dos cuidados.

1. USF Espaço Saúde
A evolução dos CSP ao longo do tempo tem sido contínua e a evidência científica a nível internacional indica que os sistemas de saúde baseados em cuidados primários efetivos, com profissionais altamente treinados e exercendo na comunidade, prestam cuidados com maior efetividade, tanto em termos de custos como em termos clínicos, em comparação com os sistemas com uma fraca orientação para os cuidados primários. ${ }^{1}$

Desta forma, as evidências demonstram que os CSP estão associados a uma distribuição mais equitativa da saúde pelas populações, com centralidade nas pessoas, 
atribuindo ao médico de família um papel de «porta de entrada» no sistema de saúde. ${ }^{2}$

Assim, o médico de família permite o contacto com o Sistema Nacional de Saúde a toda a população, garantindo-lhe acesso a cuidados de saúde, tanto a nível de consulta programada (consulta com marcação prévia) como a nível de atendimento no próprio dia (consulta não programada), sendo este tipo de consulta denominada de consulta aberta (CA). É uma consulta com critérios bem definidos, criada para o atendimento de utentes com patologia aguda ou agravamento de patologia crónica.

Segundo o documento da Missão para os Cuidados de Saúde Primários, a CA é definida como um "período de consulta com marcação presencial ou telefónica só no próprio dia". ${ }^{3}$ Com o mesmo princípio existe também nas Unidades de Saúde Familiares (USF) a CA da USF que se define, segundo este documento, como "período de consulta com marcação presencial ou telefónica só no próprio dia, para garantir a intersubstituição. Poderá não existir como período autónomo se a USF assumir a intersubstituição nos períodos personalizados de consulta individual". ${ }^{3}$

Na USF Espaço Saúde, unidade de saúde familiar no Porto, quando um utente necessita de atendimento no próprio dia, sem possibilidade de marcação de consulta programada, pode recorrer à CA. É uma consulta de iniciativa do utente, que funciona diariamente entre as $8 \mathrm{~h} 00$ e as $20 \mathrm{~h} 00$, com duração de 15 minutos, permitindo a resposta a situações agudas.

No entanto, em Portugal, à semelhança do que acontece em muitos outros países, há pouca informação sobre os motivos que levam os utentes a dirigir-se aos CSP, nomeadamente em situações agudas. Sabe-se também que muitas vezes os doentes apresentam queixas que resultam de uma panóplia complexa de elementos físicos, psicológicos e sociais, ${ }^{4}$ o que leva os utentes a recorrer inúmeras vezes aos serviços de saúde, tornando mais complexa a avaliação dos motivos da sua recorrência aos serviços de saúde.

Neste sentido, os profissionais da USF Espaço Saúde consideram que o conhecimento dos motivos de CA e do padrão de utilização dos utentes poderá ter um impacto significativo na eficiência da atuação médica e na dinâmica de trabalho da USF, o que se traduzirá em ganhos em saúde para os utentes.
Este estudo teve como principal objetivo caracterizar o perfil de CA da USF Espaço Saúde durante os meses de janeiro, abril e julho de 2014. Para tal procedeu-se à identificação e quantificação dos principais diagnósticos segundo a codificação em CSP, a International Classification of Primary Care, $2^{\text {nd }}$ edition (ICPC-2), à avaliação da frequência de intersubstituição médica e à frequência de referenciação para o serviço de urgência (SU) e outras especialidades hospitalares.

\section{MÉTODOS}

Tratou-se de um estudo descritivo (retrospetivo), observacional e transversal realizado na USF Espaço Saúde pertencente ao Agrupamento de Centros de Saúde (ACeS) Porto Ocidental.

Foram obtidos dados correspondentes aos meses de janeiro, abril e julho de 2014 e a população estudada englobou todos os utentes que recorreram à CA da USF Espaço Saúde durante o período de estudo. Não foram estabelecidos critérios de exclusão.

O protocolo do estudo foi apresentado em reunião médica e obteve aprovação dos médicos especialistas e da coordenadora da USF Espaço Saúde. Foi posteriormente submetido à Comissão de Ética da Administração Regional de Saúde (ARS) do Norte, obtendo parecer favorável, e garantida a autorização do Diretor Executivo do ACeS Porto Ocidental.

Para o estudo foram definidas variáveis qualitativas (género, escolaridade, situação laboral, isenção de taxa moderadora, patologias crónicas, médico que realizou a consulta, médico de família do utente, motivo da consulta, diagnóstico, referenciação para o SU para especialidades hospitalares [código 67 segundo a ICPC-2, International Classification of Primary Care, $2^{\text {nd }}$ edition], data da consulta, dia da semana da consulta) e quantitativas (idade, número de consultas no último ano 2014).

As informações foram recolhidas através do processo clínico eletrónico SClínico ${ }^{\circledR}$ e do Módulo de Informação e Monitorização das Unidades Funcionais (MIM@UF®); os dados obtidos foram codificados e introduzidos em base de dados construída para o efeito, com recurso ao programa Microsoft Office Excel 2015®. Para a colheita de dados foi atribuído um médico especialista a cada investigador e, de forma a garantir o anonimato dos médicos, estes foram codificados com 
letras de A a E por um investigador, o qual não efetuou colheita de dados. Todos os investigadores fizeram a recolha de dados nas instalações da USF Espaço Saúde fora do seu horário de trabalho.

Foi estudada cada variável. Nas variáveis contínuas procedeu-se ao cálculo de média e desvio-padrão para as distribuições normais e mediana para as distribuições assimétricas. Para as variáveis qualitativas obtiveram-se tabelas de frequência e foi realizada a sua representação gráfica através de gráficos de barras.

A análise estatística dos dados foi realizada através do programa Microsoft Excel 2015®.

\section{RESULTADOS}

No decorrer do período estudado foram realizadas um total de 1.310 CA na USF Espaço Saúde. O número total de consultas presenciais efetuadas neste período foi de 3.182 consultas, representando as CA $41,2 \%$ do total destas consultas ( $45 \%$ em janeiro, $38 \%$ em abril e $39 \%$ em julho).

Dos três meses em estudo, o mês de janeiro foi o que registou um maior número de CA (38,9\% em Janeiro, $n=509$ ) (Quadro I).

No que concerne ao dia da semana, a quinta-feira foi o dia com um maior número de CA $(24,0 \%, n=314)$, seguida da segunda-feira $(23,7 \%, n=310)$ e da terça-feira $(20,6 \%, n=270)$, não sendo esta diferença, no entanto, significativa.

Através da consulta da plataforma MIMUF® contabilizou-se um total de 8.605 utentes dos cinco médicos da unidade. O médico com menor número de utentes foi o médico B (19,77\%; $n=1.701)$, enquanto o médico $\mathrm{D}$ foi o que apresentou um maior número de utentes (20,31\%; $n=1.748)$.

Em relação às características demográficas da população incluída no estudo (Quadro II) verificou-se que a maioria dos utentes pertencia ao sexo feminino $(62,7 \%, n=821)$ e com uma média de idade de 44,8 anos (mediana=46,0 anos; variação entre dois e 94 anos). Quando comparados os dados prévios com os da população total inscrita na USF no ano em estudo, é concordante a predominância do sexo feminino $(51,82 \%$; $n=4.312$ ).

Na análise da situação profissional, a maioria das CA foram realizadas a utentes que estavam no ativo $(41,5 \%$, $n=544$ ), seguidas das consultas a utentes desemprega-

\begin{tabular}{|c|c|c|c|c|}
\hline \multicolumn{5}{|c|}{$\begin{array}{l}\text { QUADRO I. Número total de consultas abertas por } \\
\text { médico e mês de realização }(n=1.310)\end{array}$} \\
\hline \multirow[b]{2}{*}{ Médico } & \multicolumn{3}{|c|}{ Mês ( $n)$} & \multirow[b]{2}{*}{ TOTAL, $n$ (\%) } \\
\hline & Janeiro & Abril & Julho & \\
\hline A & 97 & 78 & 77 & $252(19,2)$ \\
\hline B & 89 & 73 & 39 & $201(15,3)$ \\
\hline $\mathrm{C}$ & 124 & 105 & 92 & $321(24,5)$ \\
\hline D & 76 & 73 & 86 & $235(17,9)$ \\
\hline $\bar{E}$ & 123 & 61 & 117 & $301(23,0)$ \\
\hline $\begin{array}{l}\text { TOTAL, } \\
\text { n (\%) }\end{array}$ & $\begin{array}{r}509 \\
(38,9)\end{array}$ & $\begin{array}{r}390 \\
(29,8)\end{array}$ & $\begin{array}{r}411 \\
(31,4)\end{array}$ & $\begin{array}{r}1.310 \\
(100,0)\end{array}$ \\
\hline
\end{tabular}

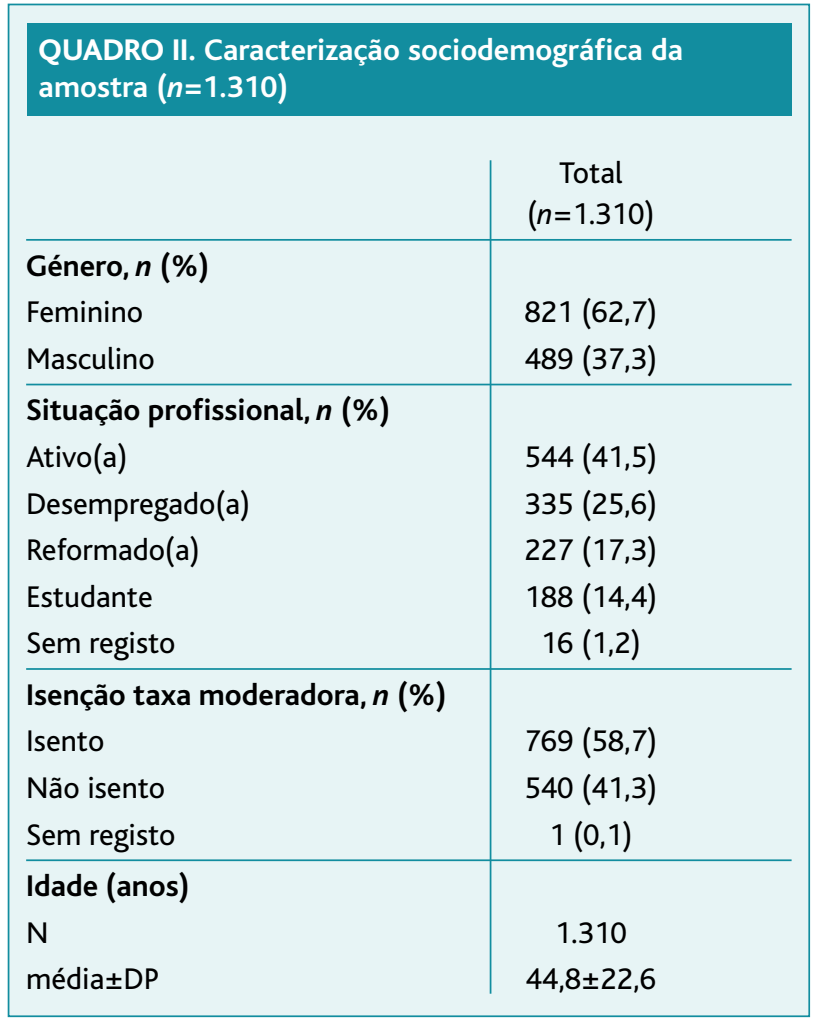

dos (25,6\%, $n=335)$, reformados $(17,3 \%, n=227)$ e, por último, aos estudantes $(14,4 \%, n=188)$. Em 16 consultas $(1,2 \%)$ não havia qualquer registo da situação profissional dos utentes.

Relativamente à isenção das taxas moderadoras verificou-se que a maioria das CA se realizaram a utentes isentos $(58,7 \%, n=769)$, não havendo registo deste 


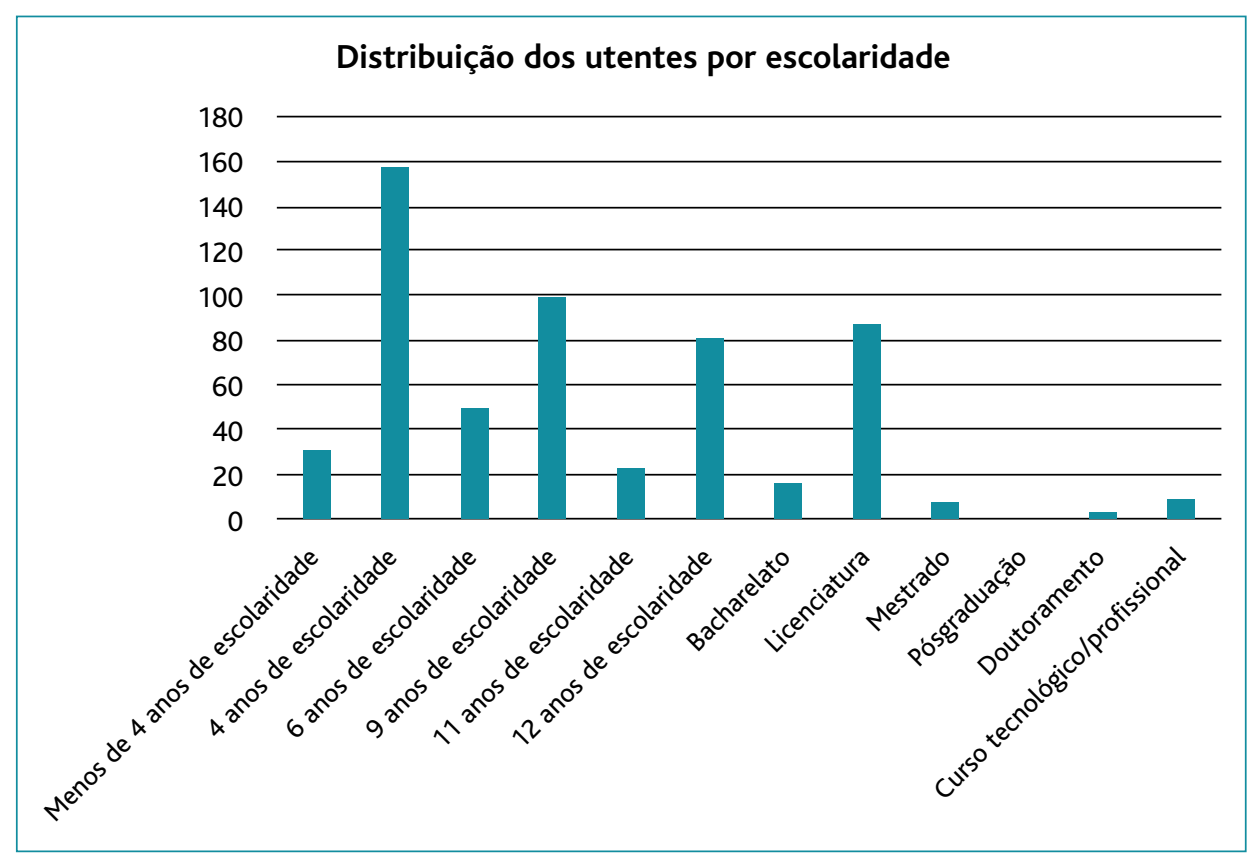

Figura 1. Distribuição dos utentes por grau de escolaridade.

parâmetro apenas numa consulta. Na análise desta variável verifica-se ainda que o médico E é o MF com uma maior percentagem de utentes isentos que recorreram à CA (34,8\% do total de isentos), seguindo-se o médico D (19,0\%) e, posteriormente, o médico C (13,3\%).

Quando analisado o grau de escolaridade no total das CA realizadas, numa grande proporção $(56,8 \%$, $n=744$ ) os utentes não tinham registo desta variável. A Figura 1 representa a distribuição dos utentes com registo desta variável por nível de escolaridade.

Dos cinco profissionais incluídos no estudo destaca-se o médico C com o maior número de CA (24,5\%; $n=321)$, seguido do médico $\mathrm{E}(22,3 \% ; n=301)$, A (19,2\%; $n=235)$, D (17,9\%; $n=235)$ e B (15,3; $n=201)$ (Quadro I).

Quando analisada a percentagem de intersubstituição, o médico B foi o que mais intersubstituíu (Quadro III), sendo que, do total de CA que realizou, 25,8\% dos utilizadores não integravam a sua lista. Já o médico que menos intersubstituíu foi o médico $\mathrm{E}$ (15\%). Os restantes médicos $\mathrm{A}, \mathrm{C}$ e $\mathrm{D}$ perfizeram um total de intersubstituição de $23,8 \%, 18,7 \%$ e $17 \%$, respetivamente. A média de intersubstituição da USF foi de 19,6\%.

Por outro lado, a lista de utentes mais utilizadora de CA foi a do médico E, com os seus utentes a consumirem um total de $371 \mathrm{CA}$, contabilizando mais de $1 / 4$ do total de CA realizadas no período estudado $(28,3 \%)$. Segue-se, por ordem decrescente de utilização destas consultas, a lista de utentes dos médicos C e D (Quadro III).

A média calculada de CA por utente no período estudado foi de 1,4 (variação = uma e nove consultas).

Adicionalmente, e no sentido de compreender os motivos que levaram cada utente a recorrer à $\mathrm{CA}$, foi feito o levantamento dos códigos ICPC-2 registados no SClínico ${ }^{\circledR}$ para cada consulta.

Perfez-se um total de 1.470 motivos de consulta, sendo que em 294 CA não houve codificação do motivo, o que corresponde a $22,4 \%$ do total de CA realizadas. A média calculada foi de 1,4 motivos por CA (variação = um e seis motivos).

Quando analisados os principais motivos pelos capítulos do ICPC-2 verificou-se, por ordem decrescente de frequência, que o capítulo A - Geral e Inespecífico foi o mais frequentemente registado $(21,16 \%)$, seguindo-se o R - Aparelho Respiratório (11,43\%) e o L - Sistema músculo-esquelético (9,52\%). Os outros capítulos registados foram por ordem decrescente: $\mathrm{D}$ - Aparelho digestivo (5,44\%), W - Gravidez, parto e planeamento familiar (4,97\%), U -Aparelho urinário (2,59\%), S - Pele $(2,52 \%), \mathrm{N}$ - Sistema neurológico $(2,04 \%), \mathrm{H}$ - Ouvido (1,77\%) e K-Aparelho circulatório (1,50\%). 


\begin{tabular}{|l|c|}
\hline \multicolumn{2}{|l|}{ QUADRO III. Número total de consultas abertas por } \\
lista de utentes e \% de intersubstituição $(n=1.310)$ \\
\\
\\
\hline Total de CA realizadas por cada médico, \\
$n$ (\%) & $1.310(100,0)$ \\
Médico A & \\
Médico B & $252(19,2)$ \\
Médico C & $201(15,3)$ \\
Médico D & $321(24,5)$ \\
Médico E & $235(17,9)$ \\
\hline Total de CA por lista de utentes, $n(\%)$ & $301(23,0)$ \\
Lista médico A & $1.310(100,0)$ \\
Lista médico B & $215(16,4)$ \\
Lista médico C & $188(14,4)$ \\
Lista médico D & $289(22,1)$ \\
Lista médico E & $233(17,8)$ \\
Sem médico de família & $371(28,3)$ \\
\hline Total de intersubstituição realizada por & $257(100,0)$ \\
cada médico, \% & \\
Médico A & $60(23,8)$ \\
Médico B & $52(25,7)$ \\
Médico C & $60(18,6)$ \\
Médico D & $40(17,0)$ \\
Médico E & $45(14,9)$ \\
\hline
\end{tabular}

Por sua vez, quando analisado especificamente o motivo (subcapítulo do ICPC-2), o mais registado de CA foi o A62 - Procedimento administrativo (6,12\%), seguindo-se o R05-Tosse (5,35\%), A31 eW31 - Exame médico/avaliação de saúde parcial (ambos com 4,97\%) (Quadro IV).

Relativamente aos diagnósticos efetuados na CA, contabilizaram-se um total de 1.718 diagnósticos, sendo que em 12 consultas não havia essa codificação. A média calculada foi de 1,3 diagnósticos por CA (variação = um e cinco diagnósticos).

Na análise dos principais diagnósticos realizados por capítulos da ICPC-2 verificou-se, por ordem decrescente de frequência, que o capítulo $\mathrm{R}$-Aparelho respiratório foi o mais frequentemente registado $(9,90 \%)$, seguido do P - Psicológico (7,34\%), do L - Sistema músculo-esquelético (6,93\%) e K-Aparelho circulatório (5,82\%).

\begin{tabular}{|c|c|}
\hline \multicolumn{2}{|c|}{$\begin{array}{l}\text { QUADRO IV. Análise comparativa de ambos os grupos } \\
\text { de inquiridos }\end{array}$} \\
\hline & $n(\%)$ \\
\hline A62 - Procedimento administrativo & $90(6,1)$ \\
\hline R05 - Tosse & $78(4,8)$ \\
\hline A31 - Exame médico/avaliação de saúde/parcial & $73(5,0)$ \\
\hline W31 - Exame médico/avaliação de saúde/parcial & $73(5,0)$ \\
\hline R21 - Sinal/ sintoma da garganta & $57(3,9)$ \\
\hline A03 - Febre & $52(3,5)$ \\
\hline A60 - Resultados de análises/procedimentos & $42(2,9)$ \\
\hline $\begin{array}{l}\text { A61 - Resultado de outro prestador - } \\
\text { exame/análises/registos/carta/procedimentos }\end{array}$ & $40(2,7)$ \\
\hline N01 - Cefaleia & $30(2,0)$ \\
\hline L03 - Sinal/sintoma da região lombar & $27(1,8)$ \\
\hline
\end{tabular}

O diagnóstico em específico mais registado foi o R74 - Infeção aguda do aparelho respiratório superior da ICPC-2 (6,52\%). Seguem-se o A98 - Medicina preventiva/Manutenção da saúde (5,71\%) e K86 - Hipertensão sem complicações $(5,18 \%)$ (Quadro V).

No que respeita aos antecedentes de saúde dos utentes que recorreram à CA no período em estudo contabilizou-se um total de 2.940 comorbilidades. Uma média de 2,2 comorbilidades por CA (variação = uma e 20 comorbilidades). Destacam-se as cinco principais que, no seu conjunto, perfazem $50,2 \%$ do total. Por ordem decrescente de prevalência: P17 - Abuso do tabaco ( $n=357,12,1 \%)$, P76 - Perturbação depressiva $(n=317$, 10,8\%), K86 - Hipertensão sem complicações ( $n=314$, $10,7 \%)$, T93 - Alterações do metabolismo dos lípidos $(n=311,10,6 \%)$ e P06 - Perturbação do sono $(n=178$, $6,1 \%)$.

Das 1.310 consultas realizadas, em apenas 22 casos $(1,9 \%)$ foi feita referenciação dos utentes ao serviço de urgência hospitalar.

Contabilizou-se ainda um total de 78 referenciações para a consulta externa hospitalar $(6,0 \%)$. Destas, o capítulo mais frequentemente registado como motivo de consulta foi o L-Sistema músculo-esquelético, que só por si constituiu uma grande porção de todos os moti$\operatorname{vos}(30,3 \%)$. Neste grupo, quando analisados os motivos em específico da CA, os três principais motivos foram: L01 - Sinal/sintoma do pescoço, L08 - Sinal/sintoma ombro e o W31 - Exame médico/avaliação de 
saúde/parcial. No entanto, tal como na análise previamente realizada, também no caso das referenciações à consulta hospitalar o motivo muitas vezes não é codificado (30\%). Por sua vez, no que respeita aos diagnósticos específicos, os três mais frequentes neste grupo foram: K86 - Hipertensão sem complicações, P76 - Perturbação depressiva e o L08 - Sinal/sintoma ombro.

\section{DISCUSSÃO}

Este estudo teve como principal objetivo caracterizar o perfil das CA da USF Espaço Saúde, nomeadamente no que diz respeito às características dos utentes que recorrem a esta consulta, aos motivos pelos quais geralmente o fazem, bem como avaliar a capacidade de resposta da USF perante condições clínicas agudas.

Em destaque, e pelos resultados obtidos, facilmente se concluiu que a percentagem de CA realizadas no período estudado $(41,2 \%)$ face ao total de consultas presenciais realizadas foi bastante superior ao expectável como objetivo a realizar em USF com características semelhantes. Perante este valor significativo, superior a $1 / 3$ do total das consultas programadas, tornou-se essencial analisar o perfil dos utilizadores, a avaliação no atendimento médico das $\mathrm{CA}$, bem como averiguar os motivos para a sua procura. No entanto, importa realçar que esta percentagem diz respeito apenas aos meses estudados (janeiro, abril e julho), pelo que uma variação sazonal limita a extrapolação com validade da percentagem de CA ao longo do ano.

Segundo as características demográficas da população, a média de idades de 44,8 anos, abrangida na população ativa, poderá dever-se à menor disponibilidade por parte dos utentes em recorrer à consulta programada nos horários disponíveis. A distribuição dos sexos, com um predomínio do sexo feminino, pode estar relacionada com dois fatores: por um lado, com o facto de as mulheres procurarem mais os cuidados de saúde; por outro lado, verifica-se que uma das principais comorbilidades mais frequentemente verificadas na CA foi a P76 - Perturbação depressiva que, como se sabe, é mais frequente no sexo feminino. ${ }^{5}$

Salienta-se ainda que, apesar do maior número de utilizadores estar no ativo (25,6\%), a maioria dos utilizadores de CA estão isentos de taxa moderadora, con-
QUADRO V. Dez principais diagnósticos realizados na consulta aberta por ICPC-2

\begin{tabular}{l|c} 
& $n(\%)$ \\
\hline R74 - Infeção aguda do aparelho respiratório superior & $112(6,5)$ \\
A98 - Medicina preventiva/Manutenção da saúde & $98(5,7)$ \\
K86 - Hipertensão sem complicações & $89(5,2)$ \\
W14 - Contraceção, outros & $54(3,1)$ \\
P76 - Perturbação depressiva & $52(3,0)$ \\
D73 - Gastroenterite, presumível infeção & $36(2,1)$ \\
T90 - Diabetes não insulino-dependente & $36(2,1)$ \\
L86 - Síndroma da coluna com irradiação de dores & $34(2,0)$ \\
U71 - Cistite/outra infeção urinária & $34(2,0)$ \\
H71 - Otite média aguda/meringite & $32(1,9)$
\end{tabular}

tribuindo como fator facilitador no que diz respeito ao acesso a este tipo de consulta.

Dos meses estudados, o de janeiro foi o que apresentou um maior número de CA. Estes resultados poderão dever-se ao perfil sazonal das infeções do sistema respiratório, mais prevalentes nos meses de Inverno, o que vai de encontro ao diagnóstico mais registado em CA (R74).

Dos cinco profissionais médicos incluídos no estudo, o que apresentou uma lista menor de utentes no ano em que decorreu o estudo foi o médico B. No entanto, a diferença entre este e o do profissional que apresenta mais utentes (médico D) perfaz um total de 47 utentes $(0,55 \%)$, pelo que se conclui que a divergência no número de CA entre os profissionais poderá ser explicada por diferentes comportamentos dos seus utentes e não pelo seu número absoluto. Por outro lado, o médico B teve uma ausência programada durante o mês de julho, o que poderá também explicar um menor número de consultas realizadas por este médico durante este mês.

Considerando que os utentes isentos representam a maioria dos utilizadores da CA $(58,7 \%)$ e o facto de o médico E ser o médico de família de $34,85 \%$ do total de utentes isentos que recorrem à CA (número que é bastante superior ao dos restantes profissionais), poderá, em parte, explicar o facto de ser um dos profissionais que realiza mais CA.

Relativamente à intersubstituição entre profissionais médicos, o destaque do médico $\mathrm{B}$, poderá ser explica- 
do pela percentagem de utilizadores das CA de cada profissional. Sendo os utentes deste médico os menos utilizadores, isto é, os que menos recorrem a CA (14,35\%), este facto poderá contribuir para a maior disponibilidade por parte deste médico. Por sua vez, o facto de o médico $\mathrm{E}$ ser o profissional que menos intersubstitui corrobora esta afirmação, particularmente tendo em conta que os seus utentes são os mais utilizadores das CA (28,32\%).

De forma a compreender com maior precisão se os resultados relativamente à população que recorre à CA se devem a questões comportamentais dos utentes ou se são simplesmente representativos da população global da USF seria necessário complementar este estudo com os dados de um ano, relativos não apenas à CA, mas também às outras consultas presenciais. Adicionalmente seria importante a realização de estudos semelhantes ao presente estudo, com foco na caracterização da CA em outras USF do mesmo ACeS, com o intuito de comparar resultados e melhorar atitudes.

Relativamente aos motivos pelos quais os utentes da USF Espaço Saúde recorrem à CA, verificou-se que em $22,4 \%$ das consultas não foi efetuado o registo deste dado, pelo que as conclusões efetuadas podem ser alvo de viés. Estes dados permitem salientar a urgência da adoção de uma codificação mais eficaz por parte da equipa médica, não só pelo seu benefício na prática clínica, mas para a realização de eventuais estudos futuros. Com os dados obtidos verificou-se, por capítulos do ICPC-2, que o A - Geral e Inespecífico foi o mais registado. Pela discriminação da lista dos 10 principais motivos de consulta enquadrados neste capítulo verificou-se que a maioria diz respeito a atividades administrativas (como a emissão de incapacidade temporária para o trabalho), atividades de avaliação do estado de saúde, pedidos de avaliação de resultados de exames complementares de diagnóstico ou mesmo para mostrar cartas de outros profissionais de saúde (ICPC-2: A62, A31, A60 e A61). Assim, foi possível verificar que muitas destas atividades estão fora do âmbito daquilo que devem ser os motivos da CA e que deverão ser antes incluídas no âmbito das consultas programadas. Nesta orientação, a informação relativa aos verdadeiros motivos de CA deverá ser reforçada a todos os utentes desta unidade.

Por outro lado, em relação aos diagnósticos mais codificados verificou-se que o capítulo $\mathrm{R}$ - Aparelho respiratório foi o mais registado $(9,90 \%)$ e, quando descriminado, foi o R74 - Infeção aguda do aparelho respiratório superior $(6,52 \%$ das CA) a codificação mais prevalente. Estes dados são consistentes com os dez principais motivos de CA dos utentes (Quadro IV) onde, excluindo os motivos específicos do capítulo A previamente mencionados, são também os mais prevalentes, associados à codificação A03 - Febre e N01 Cefaleia, sintomas que estão frequentemente relacionados com as infeções agudas do aparelho respiratório superior.

Quanto à referenciação dos utentes ao serviço de urgência verificou-se que a grande maioria dos utentes que recorre a CA $(98,1 \%)$ não necessitou de ser referenciado, concluindo-se que a USF consegue dar resposta à quase totalidade dos casos.

Por sua vez, no caso da necessidade de referenciação do utente para a consulta hospitalar, verificou-se que esta também é realizada numa pequena percentagem do total de CA (6\%). Destas, verificou-se que o capítulo L-Sistema músculo-esquelético foi o mais frequentemente registado como motivo de consulta (30\% de todos os motivos registados nestas consultas), provavelmente por se tratar de um capítulo que abrange patologias com maior cronicidade clínica (em que os utentes apresentam frequentemente necessidade de recorrer a CA devido a agudizações da dor) e também pela maior necessidade de estudo adicional através de meios-complementares de diagnóstico, o que vai de encontro aos resultados obtidos. No entanto, tendo em conta que a patologia músculo-esquelética inclui maioritariamente situações que, pela sua cronicidade, deveriam ser preferencialmente abordadas numa consulta programada, estes dados corroboram a necessidade de investir em educação para a saúde, de modo a explicar aos utentes os motivos da CA.

O diagnóstico mais registado aquando da referenciação foi o K76 - Hipertensão sem complicações. Este facto permitiu concluir que provavelmente na maioria das codificações K76 a CA serviu para abordar não só os motivos pelos quais os utentes recorreram à CA, como também outros problemas crónicos como a hipertensão arterial (codificando-o através do programa SClínicoÒ), evitando a necessidade de uma segunda deslocação à unidade. Já a codificação P76 - Perturbação depressiva, que surgiu como o segundo diagnósti- 
co mais frequente quando os utentes foram referenciados, apresenta-se mais fortemente relacionado com a dor crónica associada às patologias do aparelho músculo-esquelético.

Relativamente aos antecedentes patológicos, os utentes que mais recorreram a $\mathrm{CA}$ apresentavam como comorbilidade mais frequente o P17 - Abuso do tabaco que, por si só aumenta o risco de infeções agudas do aparelho respiratório superior (diagnóstico mais registado em CA) e está igualmente de acordo com o R05 Tosse (o segundo dos 10 principais motivos de CA). Desta forma, é fulcral e urgente a necessidade de se apostar nos incentivos à cessação tabágica, em todos os momentos de consulta, mesmo durante a CA, e a necessidade da realização de intervenção breve e/ou sugerir com maior frequência a marcação de consulta de cessação tabágica.

Este estudo permitiu concluir, em primeiro lugar, a importância de se investir na literacia em saúde dos utentes no sentido de compreenderem as indicações específicas que devem motivar a ida à CA. Os resultados do estudo reforçam ainda a importância de um correto registo clínico que, por vezes, por falta de gestão de tempo dos profissionais fica comprometido, limitando não só o seguimento clínico do utente como uma possível investigação futura. Através da análise crítica dos principais motivos/diagnósticos pelos quais os utentes recorrem à CA será possível uma melhor gestão de recursos que, aliados ao trabalho em equipa, per- mitirão uma melhor assistência e qualidade de resposta no âmbito das CA.

\section{REFERÊNCIAS BIBLIOGRÁFICAS}

1. Starfield B. Primary care: balancing health needs, services and technology. New York: Oxford University Press; 1998. ISBN 9780195125436

2. Hummers-Pradier E, Beyer M, Chevallier P, Eilat-Tsanani S, Lionis C, Peremans $L$, et al. The research agenda for general practice/family medicine and primary health care in Europe. Maastricht: European General Practice Research Network; 2009.

3. Missão para os Cuidados de Saúde Primários. Consulta aberta: consulta de agudos, não programada versus consulta aberta no hospital e centro de saúde [Internet]. Lisboa: Administração Central do Sistema de Saúde; 2007. Available from: http://www2.acss.min-saude.pt/Portals/0/Consulta_Aberta_MCSP_20071213.pdf

4. Ribeiro C. Família, saúde e doença: o que diz a investigação [Family, health and disease: what the research says?]. Rev Port Clin Geral 2007;23(3):299-306. Portuguese

5. Branco J, Gomes AA. O médico de família e a saúde mental. Lisboa: Edições Especiais; 1994.

\section{CONFLITO DE INTERESSES}

Os autores declaram não possuir quaisquer conflitos de interesse.

\section{FINANCIAMENTO DO ESTUDO}

Este estudo não foi apoiado por qualquer subsídio ou bolsa.

\section{ENDEREÇO PARA CORRESPONDÊNCIA}

Maria João Canavez Peixoto

E-mail: mariajoao.canavez@gmail.com

http://orcid.org/0000-0003-0972-290X

Recebido em 06-06-2017

Aceite para publicação em 07-10-2018 


\section{ABSTRACT}

\section{WALK-IN APPOINTMENTS: A CROSS-SECTIONAL DESCRIPTIVE ANALYSIS IN A PORTUGUESE FAMILY HEALTH UNIT}

Objectives: Walk-in Appointments (WIA) are fundamental for Primary Healthcare (PHC) and play a vital role towards the efficient management of resources, sustainability and quality of the National Health System (NHS). This study had as main objective to characterise the WIA at Espaço Saúde Family Health Unit (FHU) through the study of a wide set of variables, including reasons for encounter and diagnoses registered in each appointment.

Type of study: Cross-sectional, descriptive study

Location: Espaço Saúde FHU, ACeS Porto Ocidental, Portugal

Population: Patients observed in WIA at Espaço Saúde FHU in January, April, and July 2014.

Methods: A set of variables (age, gender, comorbidities, schooling level, work status, family physician, physician that held the appointment, exemption or non-exemption from user fees, total number of appointments that each patient had in 2014, reason for WIA, main diagnosis according to ICPC-2, and referral to the Emergency department / Hospital) were collected via the SClínico ${ }^{\circledR}$ software. Statistical analysis of the data was performed using Microsoft Excel $2015^{\circledR}$.

Results: A total of 1,310 WIA were performed, representing $41.2 \%$ of the total number of face-to-face appointments. Most patients were female $(62.7 \%, n=821)$ with an average age of 44.8 years. The most frequent users were those who were professionally active $(41.5 \%, \mathrm{n}=544)$ and exempt from user fees $(58.7 \%, \mathrm{n}=769)$. The most commonly reported reason for WIA was A62 - Administrative Procedure (6.12\%), followed by R05 - Cough (5.35\%), and by A31 and W31 - Medical Examination I Partial Health Evaluation (both at 4.97\%). The most frequent diagnosis was R74-Acute Upper Respiratory Infection (6.52\%), followed by $A 98$ - Health Maintenance / Prevention (5.71\%) and K86 - Hypertension without complications (5.18\%). The referral to emergency department occurred in only 22 appointments (1.9\%) and referral to Hospital appointment occurred in 78 appointments (6.0\%).

Conclusions: In addition to the detailed characterization of WIA at the FHU, this study highlights the importance of improving patients' health literacy, so that they can understand the specific indications that justify a WIA. The results of the study also reinforce the importance of an accurate clinical record that may often be jeopardized by clinicians' time constraints, limiting not only the adequate follow-up of patient, but also future investigations. A critical analysis of the main reasons for encounter/diagnoses observed in WIA will allow to better manage resources that, together with teamwork, will allow a better provision of care and improve the quality of service delivery in WIA.

Keywords: Walk-in Appointments; Medical inter-substitution 